\title{
High-quality Synthetic 2D Transition Metal Dichalcogenide Semiconductors
}

\author{
Dumitru Dumcenco, Dmitry Ovchinnikov, Kolyo Marinov, Andras Kis* \\ Institute of Electrical Engineering and Institute of Materials Science and Engineering, \\ École Polytechnique Fédérale de Lausanne, Lausanne, Switzerland \\ *andras.kis@epfl.ch
}

\begin{abstract}
We give here an overview on our results on the large-area growth of 2D transition metal dichalcogenide semiconductors $\mathrm{MoS}_{2}, \mathrm{MoSe}_{2}$, WSe 2 using chemical vapor deposition. The growth of $\mathrm{MoS}_{2}$ on sapphire occurs epitaxially with the crystalline orientation of the $\mathrm{MoS}_{2}$ film closely matching that of the sapphire substrate, resulting in a high-quality continuous film. The use of $\mathrm{H}_{2} \mathrm{~S}$ results in more control over growth morphologies. WSe 2 and $\mathrm{MoSe}_{2}$ have also been successfully grown using solid-state precursors. Roomtemperature mobilities of all these materials exceed $10 \mathrm{~cm}^{2} \mathrm{~V}^{-1} \mathrm{~s}^{-1}$. In contrast to $\mathrm{MoS}_{2}, \mathrm{WSe}_{2}$ and $\mathrm{MoSe}_{2}$ show ambipolar behavior.
\end{abstract}

\section{Keywords: TMDCs, large-area growth, field-effect transistors}

\section{INTRODUCTION}

The resurgence of semiconducting transition metal dichalcogenides in the context of few-layer films for mesoscopic devices [1,2] has motivated the development of scalable synthesis approaches which are necessary in order to achieve controllable thickness, size and uniformity of the 2D film. Chemical vapor deposition (CVD) is probably the most promising method for achieving this. The quality of the grown materials critically depends on the purity of the precursors but also the crystallinity of the substrate. We review here our recent work on this subject and present results which show that high-quality $\mathrm{MoS}_{2}, \mathrm{WSe}_{2}$ and $\mathrm{MoSe}_{2}$ with large grain sizes as well as optical and electronic quality approaching that of the material exfoliated from bulk crystals can be achieved.

\section{SYNTHESIS OF $\mathrm{MOS}_{2}$ USING SOLID-STATE PRECURSORS}

We have used highly polished sapphire substrates to achieve control over lattice orientation during CVD growth of monolayer $\mathrm{MoS}_{2}$, Fig. 1. [3] Even though $\mathrm{MoS}_{2}$ and sapphire interact only via the relatively weak van der Waals interaction, commensurability of the sapphire lattice with $\mathrm{MoS}_{2}$ allows the van der Waals interaction to control the lattice orientation of $\mathrm{MoS}_{2}$ in a similar way as the lattice matching requirement in covalent semiconducting materials. The growth process is based on the gas-phase reaction between $\mathrm{MoO}_{3}$ and sulphur [4,5] evaporated from solid sources using ultrahighpurity argon as the carrier gas.

This work was financially supported by Swiss SNF Sinergia Grant no. 147607, funding from the European Union's Seventh Framework Programme FP7/2007-2013 under Grant Agreement No. 318804 (SNM) and Marie Curie ITN network "MoWSeS" (grant no. 317451). We acknowledge funding by the EC under the Graphene Flagship (grant agreement no. 604391).

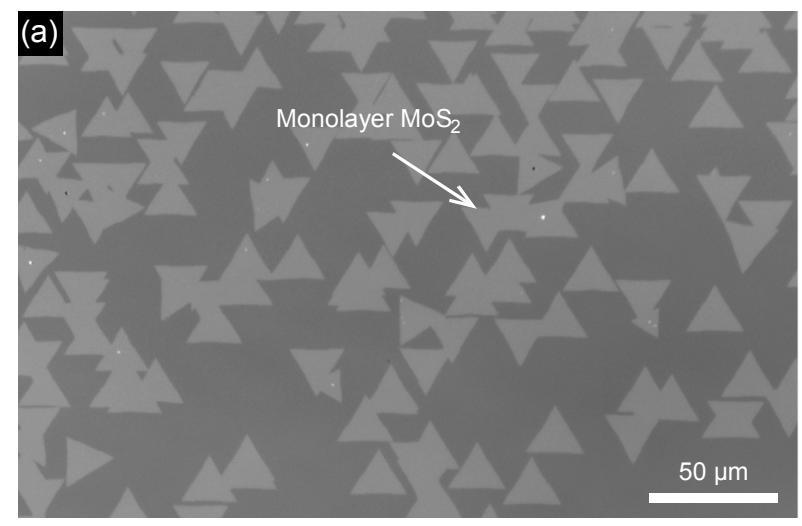

(b)

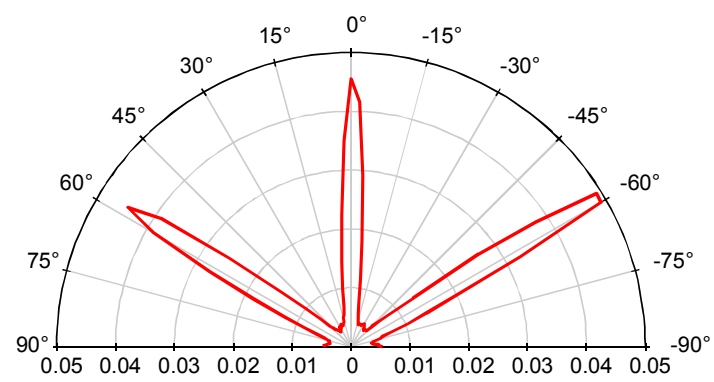

(c)

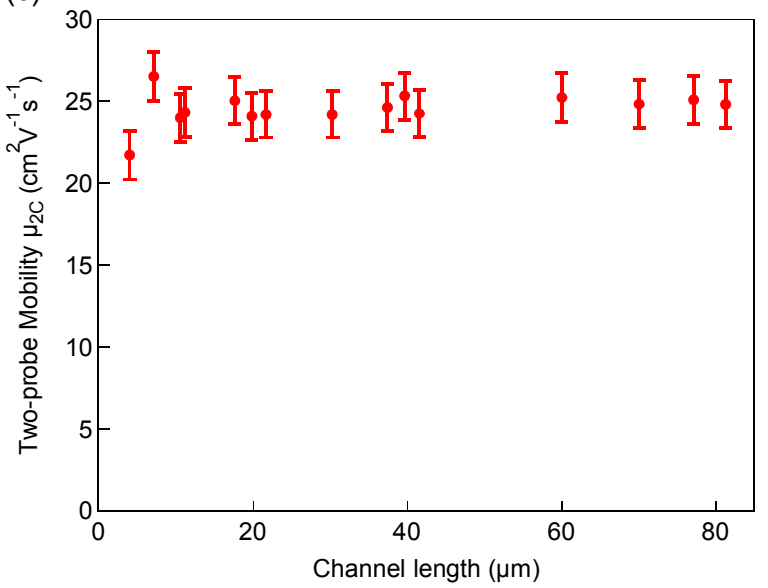

Fig. 1. (a) Optical image of triangular single-crystals of monolayer $\mathrm{MoS}_{2}$ grown on sapphire. (b) Polar plot of the orientation distribution of singlecrystal $\mathrm{MoS}_{2}$. (c) Two-probe effective field-effect mobility of a polycrystalline film as a function of channel length. 

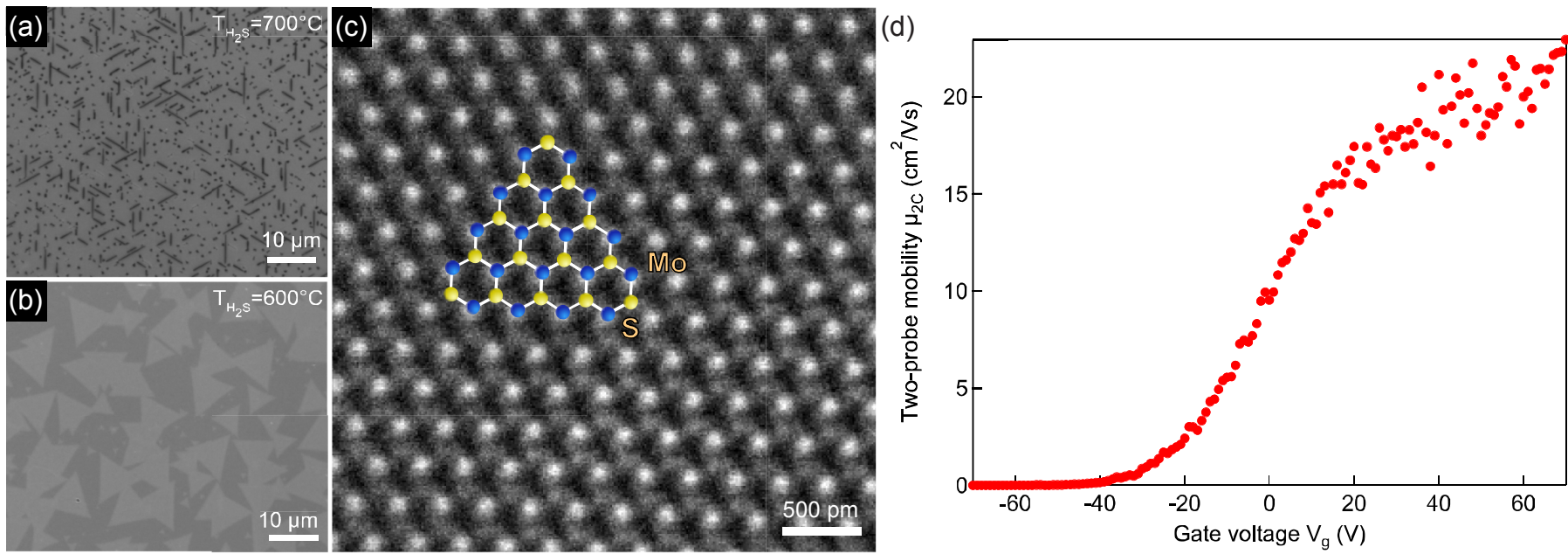

Fig. 2. (a) Growth of vertically-aligned $\mathrm{MoS}_{2}$ domains with $\mathrm{H}_{2} \mathrm{~S} / \mathrm{H}_{2}$ mixture being introduced as the sample temperature reaches $700^{\circ} \mathrm{C}$, for different $\mathrm{H}_{2} \mathrm{~S}$ and $\mathrm{H}_{2}$ flow rate of $15: 5 \mathrm{sccm}$. (b) Decreasing the injection temperature of the $\mathrm{H}_{2} \mathrm{~S} / \mathrm{H}_{2}$ mixture to $600^{\circ} \mathrm{C}$ leads to a reduction of longitudinal length of $\mathrm{MoS}_{2}$ domains, while the growth morphology changes from vertically-aligned multilayer to horizontally-aligned monolayer. (c) STEM image of single-layer $\mathrm{MoS}_{2}$ lattice with structural model overlaid, showing high-crystallinity of the grown material. (d) Two-probe field effect mobility measured on back-gated field-effect transistors based on CVD-MoS

The growth procedure results in characteristic single-crystal domains in the shape of well-defined equilateral triangles that can (depending on growth time) merge into a continuous monolayer film covering a typical area of $6 \mathrm{~mm} \times 1 \mathrm{~cm}$ in the middle portion of the growth substrate. The majority $(91.5 \%$ of islands shown on Fig. 1(a) of single-crystal domains are well aligned with the relative orientation of edges that can be expressed as multiples of $60^{\circ}$. This is confirmed by polar plot of orientations presented in Fig. 1(b), showing that the dominant edge orientations are $0^{\circ}$ and $\pm 60^{\circ}$. A small fraction of domains $(6 \%)$ show edges with a relative angle of $\pm 30^{\circ}$, while the rest $(2.5 \%)$ show other orientations.

Optical characterization (photoluminescence and Raman spectroscopy) confirm the high quality of our films. Electrical transport measurements on single grains show the mobility reach a value of $43 \mathrm{~cm}^{2} / \mathrm{Vs}$. Good stitching of such high-quality single crystals into continuous films is necessary in order to realize large-area $\mathrm{MoS}_{2}$ with the same quality as that of the single crystal. We have examined the electrical properties of polycrystalline films by performing measurements on a section of a continuous film with the length $\mathrm{L}=81.2 \mu \mathrm{m}$ and width $\mathrm{W}$ $=36.6 \mu \mathrm{m}$, contacted by multiple electrodes. The effective field-effect mobility as a function of channel length, Fig. 1(c) extracted from two-contact measurements in various lead configurations is shown on Fig. 1c. In stark contrast to previous studies by Najmaei et al. [6], where a $50 \%$ degradation in mobility was seen as the channel length was increased from $3 \mu \mathrm{m}$ to $30 \mu \mathrm{m}$, we see no degradation of the mobility with increasing channel length, with the longest $\sim 80 \mu \mathrm{m}$ long segment showing the same mobility as the shortest $(\sim 4 \mu \mathrm{m}$ long), within the measurement error. We estimate that there are $\sim 16$ junctions between single crystals along the length of the 82 $\mu \mathrm{m}$ long channel. This shows that the superior electrical properties of the single crystals are preserved over length scales larger than those corresponding to single grains.

\section{Synthesis OF $\mathrm{MOS}_{2}$ USING $\mathrm{H}_{2} \mathrm{~S}$ AS A SULPHUR SOURCE}

We have continued our work towards improving CVD growth of semiconducting TMDCs by replacing solid-state precursors with gas-phase sources which are easier to control. In this respect sulphur can be easily replaced with $\mathrm{H}_{2} \mathrm{~S}$. The growth procedure results in highly-oriented vertically-aligned multilayers or horizontally-aligned monolayer domains in the shape of well-defined equilateral triangles (Fig. 2) [7]. At a high $\mathrm{H}_{2} \mathrm{~S} / \mathrm{H}_{2}$ injection temperature of $700{ }^{\circ} \mathrm{C}$ with different $\mathrm{H}_{2} \mathrm{~S}: \mathrm{H}_{2}$ ratios $(5: 15,10: 10$ and $15: 5 \mathrm{sccm})$, vertically-aligned multilayer $\mathrm{MoS}_{2}$ domains with lengths of up to $10 \mu \mathrm{m}$ have been observed. The lateral length of $\mathrm{MoS}_{2}$ domains decreased in size to $4 \mu \mathrm{m}$ as the temperature at which the $\mathrm{H}_{2} \mathrm{~S} / \mathrm{H}_{2}$ mixture is first introduced is lowered to $625^{\circ} \mathrm{C}$. When this temperature is further reduced to $605{ }^{\circ} \mathrm{C}$, together with vertical growth, monolayer $\mathrm{MoS}_{2}$ domains start to grow in a lateral direction on the surface of the sapphire substrate. For an even lower injection temperature of $600{ }^{\circ} \mathrm{C}$, the growth is dominated by monolayer $\mathrm{MoS}_{2}$ triangles. In general, we observe that an earlier introduction of the $\mathrm{H}_{2} \mathrm{~S} / \mathrm{H}_{2}$ mixture favors horizontal over vertical growth. Moreover, by varying the ratio of $\mathrm{H}_{2} \mathrm{~S} / \mathrm{H}_{2}$ mixture, the morphology and domain size of monolayer $\mathrm{MoS}_{2}$ flakes is changed. Under more sulphidizing conditions $\left(\mathrm{H}_{2} \mathrm{~S}: \mathrm{H}_{2}=15: 5\right), \mathrm{MoS}_{2}$ domains adopt a triangular shape.

Fig. 2(c) presents the lattice of $\mathrm{MoS}_{2}$, imaged in this way and with a negligible beam-induced damage. Here, brighter spots correspond to Mo atoms and darker spots to stacks of two sulfur atoms. In general, contrast in STEM mode under these conditions of monolayer imaging is considered to obey the $Z^{1.7}$ contrast relation, which in the absence of contamination allows distinguishing between the atoms with different $\mathrm{Z}$ number or between columns with different occupancy. Extracted from Fourier-transformed STEM images and used to draw the overlaid structural model, the lattice 

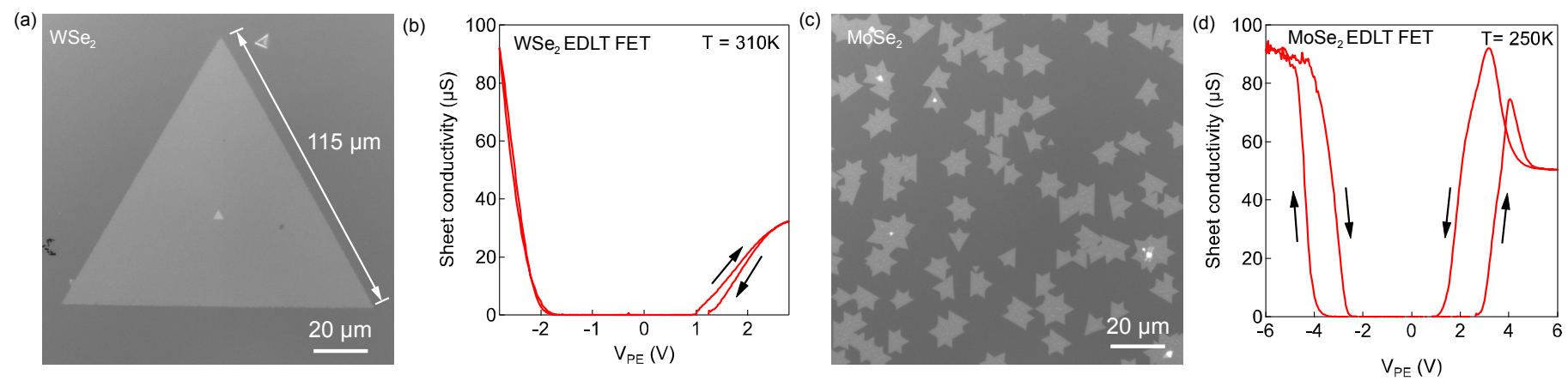

Fig. 3. (a) Optical image of monolayer $\mathrm{WSe}_{2}$ single crystal grown using CVD. (b) Transfer characteristic of a CVD-WSe ${ }_{2}$ electric double-layer field-effect transistos using ionic liquid gating. The device shows ambipolar behavior and large hole conductivity. (c) Optical image of monolayer MoSe ${ }_{2}$ crystals grown using CVD. (d) Transfer characteristic of a CVD-MoSe 2 electric double-layer field-effect transistos using ionic liquid gating.

constant of $\sim 3.18 \AA$ is in good agreement with the literature values [8].

After characterization of structural and optical properties of our material, we moved to device fabrication and electrical characterization. Material was transferred from sapphire substrates to conventional $\mathrm{Si} / \mathrm{SiO}_{2}(270 \mathrm{~nm}$ thickness $)$ and contacts were defined by standard electron beam lithography (EBL). Gold with a thickness of $90 \mathrm{~nm}$ was used as the contact material (see Methods). Some of the devices were etched in rectangular shape using the second step of EBL followed by oxygen plasma etching.

We characterized our devices in vacuum (base pressure $10^{-6}$ mbar) after annealing at $140{ }^{\circ} \mathrm{C}$ for $24 \mathrm{~h}$. We present the characteristics of a typical device after annealing on Fig. $2 \mathrm{~d}$. This device was etched in rectangular shape and was realized in a back-gated geometry using a degenerately doped $\mathrm{Si}$, covered by a $270 \mathrm{~nm}$ thick layer of $\mathrm{SiO}_{2}$. The two-probe mobility, which does not take into account the effect of contact resistance, presents the lower estimate of intrinsic properties of material. Values of $\mu_{2 \mathrm{C}} \sim 20 \mathrm{~cm}^{2} \mathrm{~V}^{-1} \mathrm{~s}^{-1}$ are in good agreement with recent reports on CVD $\mathrm{MoS}_{2}$, grown from solid precursors on $\mathrm{SiO}_{2}$ [9] and sapphire [3] substrates, which indicates a similar quality of material. We estimate an $\mathrm{I}_{\mathrm{on}} / \mathrm{I}_{\text {off }}$ ratio $\sim 10^{6}$ of this transistor at $\mathrm{V}_{\mathrm{s}}=5 \mathrm{~V}$. Overall, the device characteristics demonstrate good current injection and mobility, high $\mathrm{I}_{\text {on }} / \mathrm{I}_{\text {off }}$ ratio and electron doping of the channel, typical for exfoliated or CVD material. The observed doping levels are most probably related to sulfur vacancies and other clusters of defects, as observed in our material by STEM.

\section{SYNTHESIS OF MOSE2 AND WSE2 USING SOLID-STATE PRECURSORS}

We have also grown monolayer $\mathrm{WSe}_{2}$ and $\mathrm{MoSe}_{2}$ using solid state precursors in the form of corresponding oxides and selenium. Grain sizes over $100 \mu \mathrm{m}$ have been achieved in case of $\mathrm{WSe}_{2}$ and grains in the range of 10-50 $\mu$ min case of other TMDs. Electrical, photoluminescence and Raman characterization show properties comparable to exfoliated materials. For the purposes of electrical characterization, we have used ionic liquid gating, in order to achieve high charge densities and efficient electron and hole injection regardless of the contact material [10]. Devices are characterized in vacuum, and close to room temperature. Both CVD-grown $\mathrm{WSe}_{2}$ and $\mathrm{MoSe}_{2}$ show perfect ambipolar behavior. Both materials show room-temperature electron mobilities of $\sim 10 \mathrm{~cm}^{2} \mathrm{~V}^{-1} \mathrm{~s}^{-1}$ and low-temperature (4K) electron mobilities of $\sim 100 \mathrm{~cm}^{2} \mathrm{~V}^{-1} \mathrm{~s}^{-1}$.

\section{ACKNOWLEDGMENT}

Device fabrication was carried out in the EPFL Center for Micro/Nanotechnology (CMI). We thank Z. Benes (CMI) for technical support with e-beam lithography and D. Alexander (EPFL-CIME) and Sorin Lazar (FEI) for help with TEM imaging.

\section{REFERENCES}

[1] B. Radisavljevic, A. Radenovic, J. Brivio, V. Giacometti, and A. Kis, Nat. Nanotechnol. 6, 147 (2011).

[2] Q. H. Wang, K. Kalantar-Zadeh, A. Kis, J. N. Coleman, and M. S. Strano, Nat. Nanotechnol. 7, 699 (2012).

[3] D. Dumcenco, D. Ovchinnikov, K. Marinov, P. Lazić, M. Gibertini, N. Marzari, O. L. Sanchez, Y.-C. Kung, D. Krasnozhon, M.-W. Chen, S. Bertolazzi, P. Gillet, A. Fontcuberta i Morral, A. Radenovic, and A. Kis, ACS Nano 9, 4611 (2015).

[4] A. M. van der Zande, P. Y. Huang, D. A. Chenet, T. C. Berkelbach, Y. You, G.-H. Lee, T. F. Heinz, D. R. Reichman, D. A. Muller, and J. C. Hone, Nat. Mater. 12, 554 (2013).

[5] S. Najmaei, Z. Liu, W. Zhou, X. Zou, G. Shi, S. Lei, B. I. Yakobson, J.C. Idrobo, P. M. Ajayan, and J. Lou, Nat. Mater. 12, 754 (2013).

[6] S. Najmaei, M. Amani, M. L. Chin, Z. Liu, A. G. Birdwell, T. P. O'Regan, P. M. Ajayan, M. Dubey, and J. Lou, ACS Nano 8, 7930 (2014).

[7] D. Dumcenco, D. Ovchinnikov, O. L. Sanchez, P. Gillet, D. T. L. Alexander, Sorin Lazar, A. Radenovic, and A. Kis, 2D Mater. 2, 044005 (2015).

[8] J. Brivio, D. T. L. Alexander, and A. Kis, Nano Lett. 11, 5148 (2011).

[9] H. Schmidt, S. Wang, L. Chu, M. Toh, R. Kumar, W. Zhao, A. H. Castro Neto, J. Martin, S. Adam, B. Özyilmaz, and G. Eda, Nano Lett. 14, 1909 (2014).

[10] A. Allain and A. Kis, ACS Nano 8, 7180 (2014). 